\title{
Detección de la tendencia local del cambio de la temperatura en México
}



DOI: 10.24850 / j-tyca-2017-06-07

\section{Resumen}

Lobato-Sánchez, R., \& Altamirano-del-Carmen, M. A. (noviembre-diciembre, 2017). Detección de la tendencia local del cambio de la temperatura en México. Tecnología y Ciencias del Agua, 8(6), 101-116, DOI: 10.24850/j-tyca-2017-06-07.

Registros de temperatura indican que tres de cada cuatro estaciones climáticas evaluadas en México señalan un calentamiento en el periodo 1950-2013, tomando como referencia al periodo base 1961-1990. Después de un análisis completo de registros climáticos en México, se determinó que solamente 112 registros cumplen con la calidad y estándares requeridos para el presente estudio, de ahí se seleccionó, de forma aleatoria, un subconjunto de 20 estaciones con tendencia positiva, representativas de entornos rurales y urbanos, distribuidas en las regiones de México. Se identificó que el periodo más reciente de calentamiento intenso inicia por lo general a mediados de la década de 1970, lo cual es congruente con la señal identificada por el Panel Intergubernamental de Expertos sobre el Cambio Climático para la temperatura media global. En las zonas rurales se observa un desfase de hasta 10 años en el inicio del periodo reciente de calentamiento en comparación con zonas urbanas para una misma región del país. El calentamiento y la diferencia entre periodos cálidos y fríos son mayor, regularmente, en áreas urbanas en comparación con las rurales.

Palabras clave: temperatura, tendencias, detección, control de calidad, cambio climático.

\section{Introducción}

Los cambios en el clima de la Tierra a lo largo de su existencia pueden deberse a variaciones
Lobato-Sánchez, R., \& Altamirano-del-Carmen, M. A. (NovemberDecember, 2017). Detection of local temperature trends in Mexico. Water Technology and Sciences (in Spanish), 8(6), 101-116, DOI: 10.24850/j-tyca-2017-06-07.

Temperature records indicate that three of the four climatic stations evaluated in Mexico show warming over the years 1950 to 2013, as compared to the period 1961 to 1990 which was used as a baseline. After a complete analysis of climate records in Mexico, only 112 were determined to have met the quality and standards required for the present study. From those, a subset of 20 stations with a positive trend were randomly selected, which were representative of urban and rural areas distributed across Mexico. The mid 1970s was identified as the most recent period of intense warming, which is consistent with the mean global temperature indicated by the Intergovernmental Panel on Climate Change. A 10-year difference in the start of the warming period was found between rural and urban regions in Mexico. Warming and the difference between warm and cold periods are typically greater in urban areas than in rural areas.

Keywords: Temperature, trend, detection, quality control, climate change.

Recibido: 22/02/2016

Aceptado: 12/06/2017

en la dinámica interna del sistema del clima o bien por forzamientos externos. Los forzamientos pueden ser de origen natural, como variaciones en la actividad solar o en la actividad volcánica, 
o variaciones de origen humano, a través del aumento de emisiones de gases de efecto invernadero y cambios en el uso del suelo, entre otros factores.

Cuando no existe variación en los forzamientos externos, los cambios del clima son influenciados sólo por los procesos de la dinámica interna del sistema. De este modo se tiene conocimiento de cambios del clima de corto o largo plazos relacionados con sistemas de interacción océano-atmósfera, como en el caso de la variabilidad climática asociada con el fenómeno de El Niño-Oscilación del Sur (ENSO, por sus siglas en inglés), o de las variaciones en periodos más largos, como las vinculadas con la Oscilación Decadal del Pacífico (PDO, por sus siglas en inglés) y la Oscilación Multidecadal del Atlántico (AMO, por sus siglas en inglés), o incluso con grandes erupciones volcánicas.

A fin de identificar tendencias de cambios del clima se realizan evaluaciones de "detección" para demostrar que el clima o el sistema afectado por el clima han cambiado en un sentido estadístico sin explicar el origen o la "atribución" de dichos cambios. Es necesario realizar investigaciones detalladas adicionales para determinar la atribución a causa alguna. Un cambio identificado es detectado en observaciones instrumentales si se determina que es pequeña su probabilidad de ocurrencia debido sólo a la variabilidad interna (Hegerl et al., 2010).

De esta forma se tiene que el calentamiento global detectado en la tendencia de incremento de la temperatura media del planeta es uno de los indicadores de un cambio climático en nuestro tiempo. En informes recientes de evaluación, el Panel Intergubernamental de Expertos sobre el Cambio Climático (IPCC, por sus siglas en inglés) concluyó que "El calentamiento del sistema climático es inequívoco" (Trenberth et al., 2007). Además de registrar un calentamiento multidecadal notable, la temperatura media global en superficie muestra una variabilidad decadal e interanual considerable (IPCC, 2013, 2014).

Así, la influencia antropogénica en el clima ha sido detectada y atribuida robustamente a escala global, pero para muchas aplicaciones es más útil una estimación de la contribución antropogénica en las tendencias de temperatura en una región en particular (Hegerl et al., 2007), en este caso para México. La detección y atribución del cambio climático a escala continental o más pequeña es más difícil de detectar que a escala global debido a la mayor contribución de la variabilidad interna en escalas pequeñas en relación con la respuesta al forzante antropogénico, la mayor dificultad para distinguir entre diferentes factores que causan el cambio a escala pequeña, y errores incrementales en la representación de detalles regionales por parte de modelos del clima que omiten forzantes que pueden ser importantes a menor escala, como el cambio de uso de suelo o el carbono negro (Bindoff et al., 2013).

En el presente trabajo se detectan y analizan las tendencias positivas de la temperatura media a nivel local en diferentes regiones de México, a partir del procesamiento de series históricas de la temperatura registrada en 112 estaciones climáticas selectas para el periodo 1950-2013, con respecto al periodo base 19611990. En el presente estudio se consideró tomar en cuenta estudios climáticos en México previos, donde en mayor medida se realizaron esfuerzos sobre la calidad de la información (Sigro, 2012; Bravo, Aspra, Zarraluqui, Gay, \& Estrada, 2012; Méndez, Ramírez, Cornejo, Zarate, \& Cavazos, 2010; Pavia, Graef, \& Reyes, 2006; Englehart \& Douglas, 2002, 2004; Pieyns, 2003; Mantua \& Hare, 2002).

Las estaciones elegidas cumplieron criterios objetivos de control de calidad aplicados a 1099 estaciones identificadas como las que se han empleado en estudios climáticos para México (Bravo et al., 2012; Sigro, 2012; Méndez et al., 2010; Pieyns, 2003), a partir, la mayoría de ellas, de la base de datos climática nacional del Climate Computing Project (CLICOM), iniciativa promovida internacionalmente por la Organización Meteorológica Mundial (OMM) desde 1985 (OMM, 2015), que en México es administrada por el Servicio Meteorológico Nacional (SMN). 


\section{Datos y métodos}

\section{Área de estudio}

La región de estudio lo representa la república mexicana (México), situada en el en el hemisferio norte del continente americano; parte de su territorio se encuentra en América del Norte y el resto en América Central (INEGI, 2014). Se ubica entre los océanos Atlántico y Pacífico, y presenta variaciones importantes en el relieve topográfico. México está en la zona de transición entre la región tropical y de latitudes medias, en la que confluyen principalmente fenómenos atmosféricos como frentes fríos, ondas del este, ciclones tropicales y la interacción de la zona de convergencia intertropical (ZCIT), que induce a un régimen monzónico de lluvias concentradas en la estación de verano.

\section{Bases de datos y metodología}

La base de datos climáticos del CLICOM (OMM, 2015) utilizada, actualizada a julio de 2015, cuenta con registros de 5031 estaciones que reportaron temperatura mínima diaria y 5034 que reportaron temperatura máxima diaria, aunque el número de estaciones con información en una misma fecha es mucho menor, similar a lo encontrado por Bravo, Aspra, Zarraluqui, Gay y Estrada (2012).

Se conjuntó una relación de 2252 estaciones climáticas a partir de las utilizadas en siete investigaciones identificadas para México (cuadro 1). Los estudios considerados han evaluado regiones climáticas con características similares entre las estaciones climáticas que las representan y al mismo tiempo características diferentes entre regiones (Bravo et al., 2012; Sigro, 2012; Pieyns, 2003), así como la teleconexión de patrones climáticos en el país con oscilaciones estacionales, interanuales, decadales y multidecadales, como El Niño o la PDO, entre otros (Méndez et al., 2010).

Se cotejaron en forma cruzada los grupos de estaciones de los siete estudios, pues éstas no son excluyentes y algunas están duplicadas entre las investigaciones (cuadro 1). A partir del análisis se identifica que:

- 1099 estaciones son independientes.

- 1153 se duplican entre los grupos, lo cual implica que están contenidas como parte de las 1099 estaciones independientes.

Después de identificar el conjunto de 1099 estaciones climáticas independientes se obtuvieron y procesaron los datos diarios de las mismas para las variables de temperatura máxima $(T \mathrm{mx})$ en ${ }^{\circ} \mathrm{C}$ y temperatura mínima $(\mathrm{Tmn})$ en ${ }^{\circ} \mathrm{C}$ de la base de datos del CLICOM para México.

La temperatura media diaria se calculó como el promedio de la temperatura máxima y mínima diarias, tal como lo recomienda la Organización Meteorológica Mundial (OMM, 2011):

Cuadro 1. Resultados de la comparación de las estaciones utilizadas en estudios climáticos.

\begin{tabular}{l|c|c|c}
\hline \multicolumn{1}{c|}{ Estudio climático } & Estaciones duplicadas & Estaciones independientes & Total \\
\hline $\begin{array}{l}\text { 1. Monitor de Sequías de México } \\
\text { (MSM) }\end{array}$ & 0 & $\mathbf{3 5 8}$ & 358 \\
\hline 2. Sigro, 2012 & 276 & $\mathbf{1 0}$ & 286 \\
\hline 3. Dr. Douglas & 74 & $\mathbf{1 3 9}$ & 213 \\
\hline 4. Bravo et al., 2012 & 131 & $\mathbf{2 1 8}$ & 349 \\
\hline 5. Méndez et al., 2010 & 310 & $\mathbf{2 7 1}$ & 581 \\
\hline 6. Pieyns, 2003 & 116 & $\mathbf{5 9}$ & 175 \\
\hline 7. Análisis propio & 246 & $\mathbf{4 4}$ & 290 \\
\hline Total & $\mathbf{1 1 5 3}$ & $\mathbf{1 0 9 9}$ & $\mathbf{2 2 5 2}$ \\
\hline
\end{tabular}




$$
\text { Tmed }=\frac{T m x+T m n}{2}
$$

A partir de los datos diarios del CLICOM se calcularon valores mensuales y anuales; estos últimos corresponden a la escala temporal de análisis considerada en el artículo. El criterio considerado es que el dato mensual debe contener más del $80 \%$ de datos diarios y para calcular el dato anual se debe tener más del $80 \%$ de los meses con datos en cada año; en caso contrario se considera como dato perdido. En la figura 1 se presenta el total de años con que disponen de información de la temperatura media las 1 099 estaciones climáticas. En varias partes del país, las estaciones tienen 50 o más años de información, excepto algunas zonas de Guerrero, Chihuahua, Coahuila, Durango, Sonora,
Sinaloa y norte de la Península de Yucatán, donde las estaciones cuentan con menos de 50 años con información.

Se procesó el periodo 1950 a 2013 como el rango temporal común para el análisis de tendencias debido a que la mayoría de las series de tiempo en las estaciones climáticas contaron con información continua y con los menores vacíos a partir de 1950.

Se aplicaron criterios de control de calidad a las 1099 estaciones climáticas, con el fin de garantizar que la información posea buena calidad, distribución espacial y suficientes observaciones como se sugiere para estudios climáticos (Bravo et al., 2012; Méndez et al., 2010; Alexander et al., 2006). Los criterios aplicados para considerar las estaciones en la mayoría de los siete estudios analizados se enfocan en la

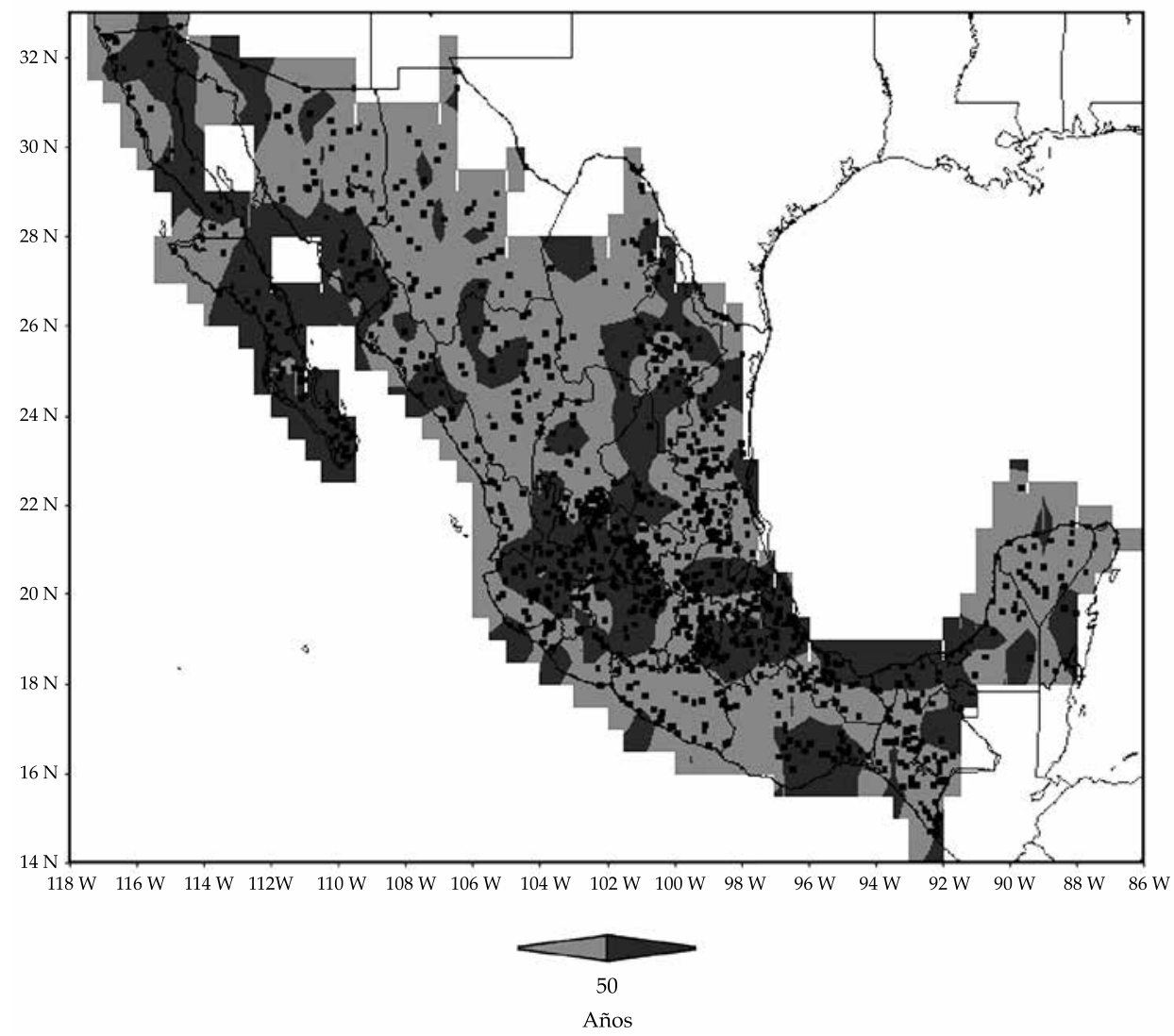

Figura 1. Representación de la cobertura temporal de información de la temperatura media de 1099 estaciones climáticas. Los puntos indican la ubicación de las estaciones. 
disponibilidad mínima de información diaria, mensual y anual en la serie.

En tanto que los criterios de control de calidad aplicados en el presente estudio (cuadro 2) son similares a los sugeridos por el Grupo de Expertos en Detección e Índices de Cambio Climático (ETCCDI, por sus siglas en inglés) coordinado por la Comisión de Climatología de la OMM (CCl/OMM) (Alexander et al., 2006), los considerados por Sigro (2012), y Méndez, Ramírez, Cornejo, Zarate y Cavazos (2010). Asimismo, si se presentan cinco o más días consecutivos con datos perdidos, se consideró el mes como dato perdido (OMM, 2011).

De la aplicación de los criterios de control de calidad a las 1099 estaciones climáticas se sustituyeron como datos perdidos 2676 datos diarios de 2820 datos sospechosos identificados (cuadro 2).

En la selección de las estaciones para la detección de la tendencia de la temperatura media local en regiones de México se aplicaron criterios objetivos presentados en el cuadro 3 . A cada criterio se le ponderó una calificación en función del grado de rigurosidad de su aplicación, siendo 3 (gris claro, denotado como gris 3) para el más riguroso y 1 (gris más oscuro, denotado como gris 1) para el más flexible. Como parte de los criterios se considera el periodo climatológico base 1961-1990 y se excluyen de los análisis anomalías para un margen de cambio de $\pm 0.25^{\circ} \mathrm{C}$, como una aproximación que considera indirectamente potenciales errores instrumentales.

El número de estaciones que cumplen la secuencia de cada uno de los cuatro criterios aplicados, agrupadas por la calificación obtenida, se muestra en la figura 2. En la aplicación secuencial de los cuatro criterios se consideraron las estaciones con las dos calificaciones más altas (gris 3 y 2).

Se realizó un análisis cualitativo de las series de anomalía de la temperatura media de las 383 estaciones que cumplieron los cuatro criterios, con el fin de realizar una evaluación más profunda de las estaciones que no presentaran lo siguiente: secuencias de tres o más años vacíos en la parte intermedia de las series, ausencia de

Cuadro 2. Criterios de control de calidad considerados y resultados de su aplicación.

\begin{tabular}{l|r|r}
\multicolumn{1}{c|}{ Criterio } & Datos sospechosos identificados & Datos sustituidos \\
\hline Temperatura mínima es mayor o igual que la máxima & 2524 & 2524 \\
\hline $\begin{array}{l}\text { Diferencia mayor que } 20{ }^{\circ} \mathrm{C} \text { entre la temperatura mínima y } \\
\text { máxima, en combinación con una: }\end{array}$ & \multicolumn{2}{|c}{} \\
\hline $\begin{array}{l}\text { 1. Diferencia interdiaria mayor o igual que } 20^{\circ} \mathrm{C} \text { con respecto a } \\
\text { los dos días adyacentes }\end{array}$ & 170 & 140 \\
\hline $\begin{array}{l}\text { 2. Diferencia interdiaria mayor o igual que } 25^{\circ} \mathrm{C} \text { con respecto a } \\
\text { uno de los dos días adyacentes }\end{array}$ & 126 & 12 \\
\hline Total & $\mathbf{2 8 2 0}$ & $\mathbf{2 6 7 6}$ \\
\hline
\end{tabular}

Cuadro 3. Criterios aplicados para la selección de estaciones a considerar en el análisis de tendencias.

\begin{tabular}{l|l|l|l|l}
\hline Criterio/semáforo & $\begin{array}{c}\text { 1. Longitud temporal } \\
\text { de la serie }\end{array}$ & 2. Año final de la serie & $\begin{array}{c}\text { 3. Disponibilidad de } \\
\text { información anual en } \\
\text { el periodo 1961-1990 }\end{array}$ & $\begin{array}{c}\text { 4. Anomalía con } \\
\text { respecto al periodo } \\
\mathbf{1 9 6 1 - 1 9 9 0}\end{array}$ \\
\hline 3. Gris 3 & $\begin{array}{l}\text { Igual o mayor que } 60 \\
\text { años }\end{array}$ & Igual o mayor a 2010 & $100 \%$ de los años & Mayor que $0.25^{\circ} \mathrm{C}$ \\
\hline 2. Gris 2 & Entre 50 y 60 años & Entre 2000 y 2010 & $\begin{array}{l}\text { Entre } 90 \% \text { y menor a } \\
100 \%\end{array}$ & Menor que $-0.25^{\circ} \mathrm{C}$ \\
\hline 1. Gris $\mathbf{1}$ & Menor que 50 años & Menor a 2000 & $\begin{array}{l}\text { Menor a } 90 \% \text { de los } \\
\text { años }\end{array}$ & Entre -0.25 y $0.25^{\circ} \mathrm{C}$ \\
\hline
\end{tabular}




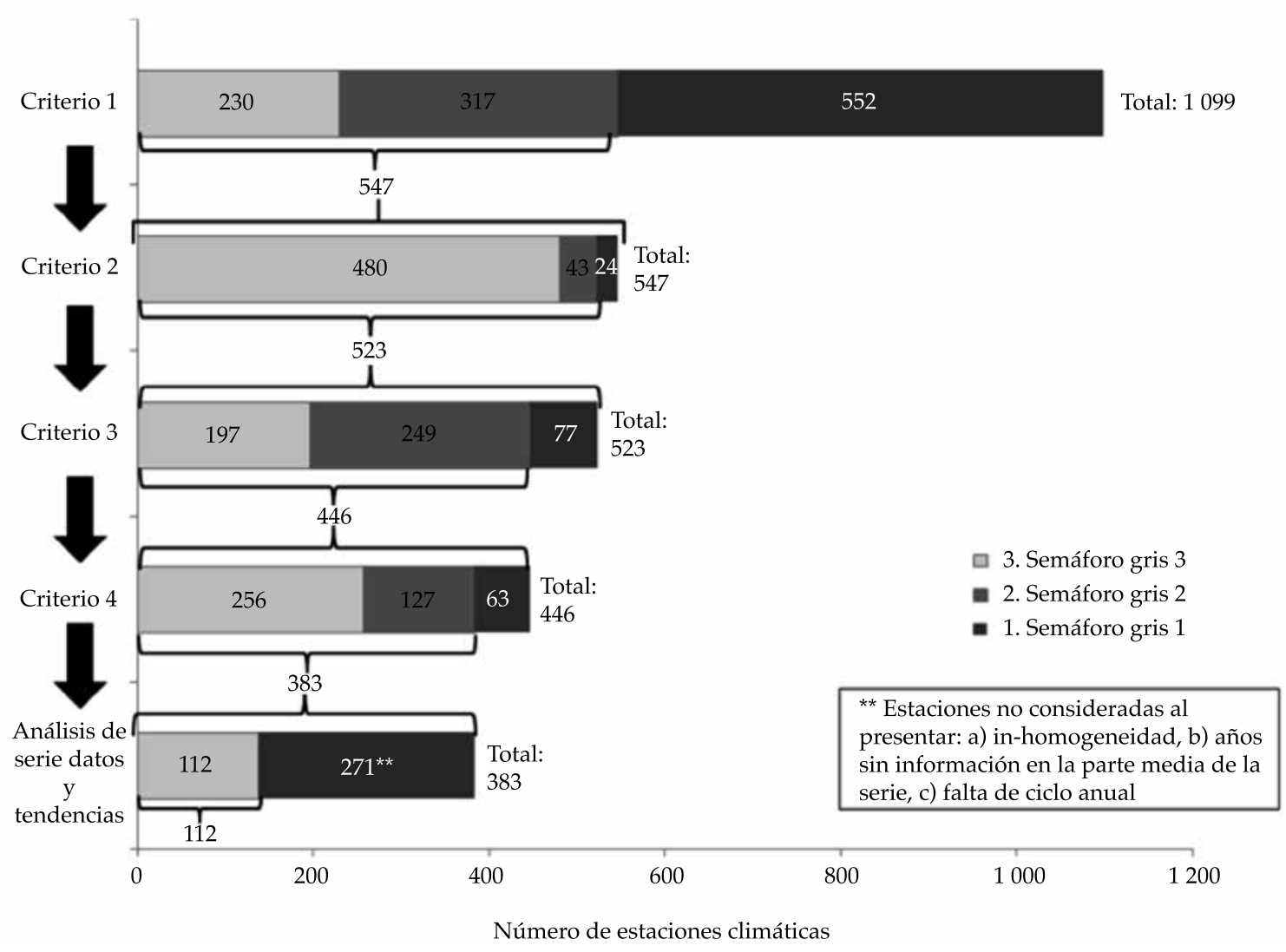

Figura 2. Total de estaciones agrupadas por calificación (o semáforo) obtenida durante la aplicación secuencial de los cuatro criterios de selección y de la revisión de las gráficas de las series de anomalías.

ciclo anual y situaciones explícitas de inhomogeneidad. Posterior al análisis, se identificaron 112 estaciones climáticas para evaluar la tendencia de la temperatura local en México.

\section{Resultados}

De los registros históricos analizados se tiene que 112 estaciones climáticas cumplen con la calidad y estándares requeridos para el presente estudio; de éstas, 84 corresponden a anomalías positivas y 28 a negativas (figura 3); en esta proporción, 3 de cada 4 estaciones señalan un calentamiento, en comparación con 1 de cada 4 , que señala un enfriamiento. Este hallazgo coincide con los publicados por el IPCC (2013) en el sentido que la Tierra experimenta un proceso de calentamiento global donde algunas regiones muestran diferentes condiciones o niveles de calentamiento, o incluso condiciones de enfriamiento en otras.

De la figura 3 se observa que las estaciones con señal de calentamiento y enfriamiento están intercaladas, resultados consistentes con lo presentado por Hartmann et al. (2013) para estaciones climáticas de no sólo los países vecinos, sino para todo el orbe. Lo anterior probablemente esté relacionado con factores como el entorno urbano/rural de las estaciones, diferencias en altitud, patrones físicos locales del clima o el mantenimiento a la infraestructura de medición.

El 48.8\% de las estaciones con señal de calentamiento presenta un incremento moderado menor a $1{ }^{\circ} \mathrm{C} ; 41.7 \%$ de incremento alto, con valores entre 1 y $2{ }^{\circ} \mathrm{C}$; y el $9.5 \%$ de incremento muy alto, 


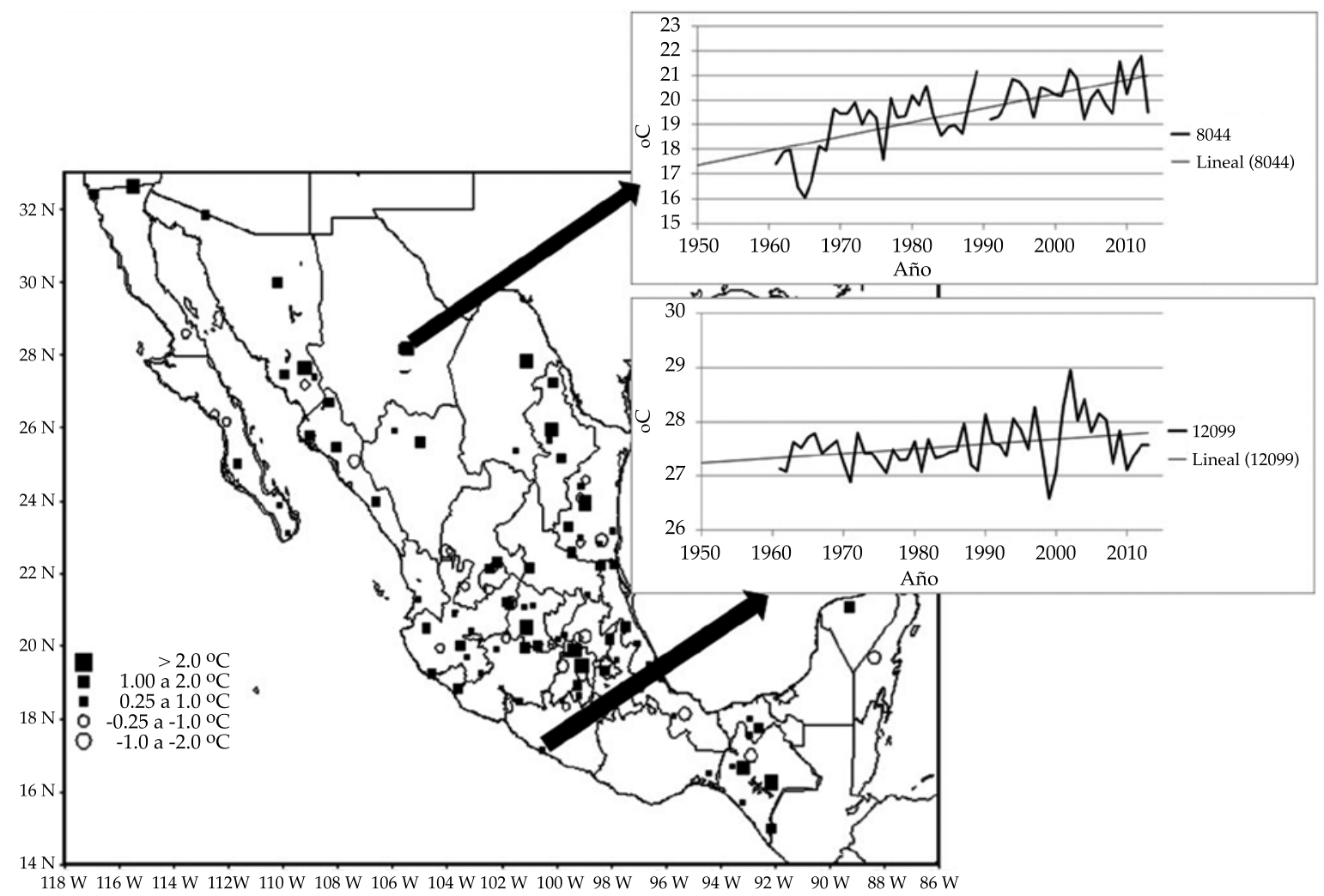

Figura 3. Distribución de estaciones de acuerdo con la intensidad de la anomalía (a) positiva (cuadros rellenos) y (b) negativa (círculos sin rellenar), con respecto al periodo base 1961-1990. Se incluye un gráfico de la serie anual con baja influencia continental (estación 12099) y alta influencia (estación 08044).

con valores mayores a $2{ }^{\circ} \mathrm{C}$. De las estaciones que indican enfriamiento: $53.6 \%$ presenta un decremento moderado mayor que $-1{ }^{\circ} \mathrm{C}$ y $46.4 \%$ con un decremento alto, con valores entre -1 y $-2{ }^{\circ} \mathrm{C}$. Destaca que no se identificaron estaciones con anomalías de muy bajo decremento, menor que $-2{ }^{\circ} \mathrm{C}$.

Con el objeto de confirmar la hipótesis inicial, en el sentido de que la tendencia positiva de cambio climático está presente tanto en zonas urbanas como rurales, a partir de las 84 estaciones climáticas identificadas con tendencia positiva se consideraron 20 estaciones selectas para el análisis de detección de tendencias de calentamiento. Las estaciones se seleccionaron en función de la distribución espacial, la elevación en el país, la longitud temporal de las series y el entorno ambiental actual en el que se encuentran ubicadas: a) urbano, con población mayor a 100000 habitantes o b) rural con población menor a 100000 habitantes en el año 2010 (figura 4).

Se consideró la población para el año 1980 y 2010 en los municipios urbanos (recuadro en la figura 4), con el fin de inferir la potencial contribución de la isla de calor urbana a la señal del calentamiento local. El IPCC señala que es improbable que el efecto de isla de calor urbana y el cambio de uso de suelo hayan contribuido en más del $10 \%$ de la tendencia de la temperatura media global de la superficie. Sin embargo, en algunas regiones con rápido desarrollo, los impactos de la isla de calor urbana y el cambio de uso de suelo pueden ser sustancialmente elevados en las tendencias regionales de temperatura (Bindoff et al., 2013). 


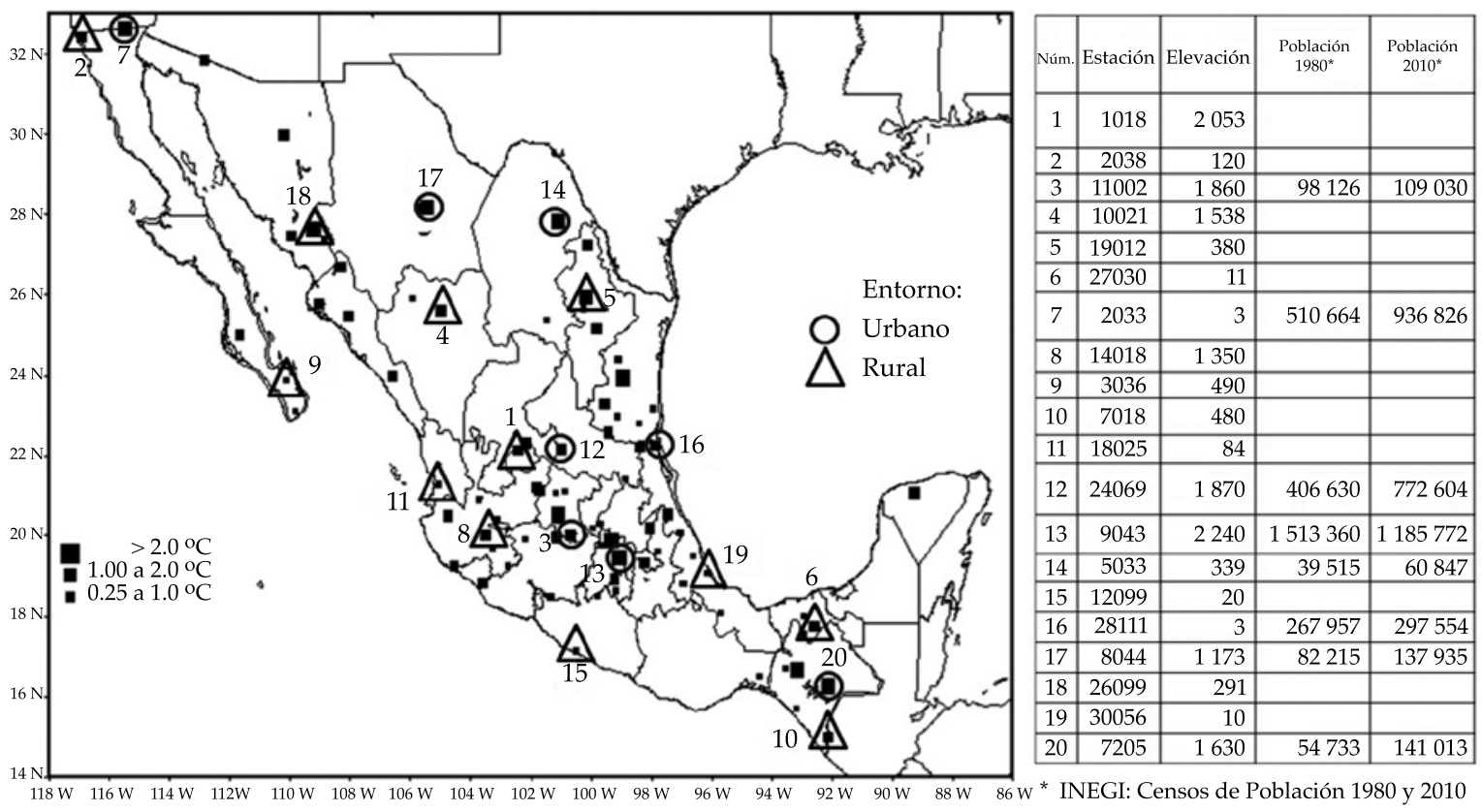

Figura 4. Representación de 20 estaciones selectas para el análisis de tendencia positiva de temperatura. Los números se asocian con los de la primera columna del recuadro.

A las 20 estaciones climáticas selectas se les aplicó un análisis de homogeneidad, complementario al control de calidad, mediante la herramienta "R-Standard Normal Homogeneity Test-rsnht.r" (Aguilar, 2010) para la detección y corrección de "brincos" en la serie de datos mensuales, relacionados con el cambio de instrumento de medición por desperfecto, cambio de observador o de ubicación de la estación (y con ello el entorno), e inconsistencia en las series, validados con respecto a los registros en papel (metadatos) del acervo histórico del SMN.

Asimismo, y con el fin de identificar la potencial influencia de las características continentales sobre la tendencia de la temperatura de las 20 estaciones selectas se aplicó un índice de continentalidad termo-pluviométrica de Gorezyñski-Valle Melendo (del Valle, 1991):

$$
\mathrm{J}=\left(\frac{1.7 * \mathrm{~A}}{\operatorname{Sen}(\text { Lat })}-20.4\right) * \frac{\% \text { P.V. }}{\% \text { P.I. }}
$$

Donde A se refiere a la amplitud térmica (diferencia entre la temperatura media del mes más cálido y la del mes más frío, en ${ }^{\circ} \mathrm{C}$ ); \% P.V. es el porcentaje de la precipitación acumulada de junio, julio y agosto, con respecto al acumulado anual; \%P.I. es el porcentaje de la precipitación acumulada de diciembre, enero y febrero con respecto al acumulado anual; Lat es la latitud geográfica de la estación climática en radianes.

A partir de los resultados, se encontró que es reducida la variabilidad interanual de la temperatura en estaciones con valores bajos del índice (cuadro 4) y ésta aumenta en estaciones que presentan mayor influencia continental, no obstante que algunas se encuentren en zonas costeras. Un rasgo interesante de continentalidad obedece al tipo de sistemas atmosféricos que inciden en la región y al patrón estacional de la precipitación donde está la estación climática. Por ejemplo, los valores del índice de continentalidad termo-pluviométrica en las estaciones ubicadas en la vertiente del Golfo de México muestran valores más altos que aquellos 
Lobato-Sánchez \& Altamirano-del-Carmen, Detección de la tendencia local del cambio de la temperatura en México

Cuadro 4. Resultados del índice de continentalidad para las estaciones selectas.

\begin{tabular}{c|c|c|c}
\hline Estación & Índice & Estación & Índice \\
\hline 7018 & -166.9 & 28111 & 129.3 \\
\hline 12099 & -142.7 & 19012 & 132.4 \\
\hline 2038 & 0.3 & 1018 & 151.9 \\
\hline 2033 & 23.2 & 26099 & 157.2 \\
\hline 27030 & 25.3 & 9043 & 179.2 \\
\hline 7205 & 74.4 & 5033 & 192.2 \\
\hline 3036 & 81.9 & 30056 & 267.5 \\
\hline 24069 & 92.6 & 8044 & 288.3 \\
\hline 14018 & 115.5 & 11002 & 305.9 \\
\hline 18025 & 127.0 & 10021 & 413.2 \\
\hline
\end{tabular}

de la vertiente del Pacífico, sin que esto sea una regla. La interpretación de estos valores es que la vertiente del Golfo de México está influenciada por sistemas meteorológicos de tipo continental, como frentes fríos, y se da una reducción de precipitación en la temporada de lluvia, conocida como canícula, situaciones que ocurren con menos regularidad en el Pacífico.

La señal de calentamiento por influencia antropogénica está presente en la tendencia positiva de las series de la temperatura de las 20 estaciones climáticas, con independencia del grado de influencia continental en las estaciones; no obstante, la intensidad del calentamiento es mayor en estaciones del centro y norte del país (recuadros en la figura 3), con alta influencia continental. Estos hallazgos son congruentes con las proyecciones de cambio climático para las próximas décadas, los cuales señalan que los mayores cambios de temperatura en México se presentarán hacia el norte del país, siendo de alta intensidad en el interior del continente (Tercera y Cuarta Comunicación Nacional de México ante la Convención Marco de las Naciones Unidas sobre el Cambio Climático) (Semarnat-INE, 2006 y 2009).

Las series de tiempo de la temperatura media en las 20 estaciones presentan variaciones caracterizadas por periodos fríos y cálidos intercalados que responden a la influencia, entre otros, de oscilaciones como El ENSO, la PDO y la $\mathrm{AMO}$, la topografía, las variaciones en tipos de vegetación y uso de suelo, cercanía con el mar, fenómenos físicos como el desplazamiento latitudinal de la ZCIT, la actividad interanual de los ciclones tropicales y del monzón de Norteamérica, así como la actividad de la corriente en chorro, los frentes fríos y los sistemas convectivos de mesoescala (Bravo et al., 2012; Méndez et al., 2010; Englehart \& Douglas, 2002, 2004).

En las figuras 5 y 6 se presentan las gráficas de las series de la temperatura y su tendencia para estaciones de caso, con respecto al periodo base 1961-1990. Del análisis de las series se identifica que:

- El periodo más reciente de calentamiento intenso inicia por lo general a mediados de la década de 1970 en estaciones con entorno urbano que se ubican en el norte y centro del país, y a principios de los años 1980 en áreas urbanas del Golfo de México y sur del país (figura 5), lo cual es congruente con la señal identificada por el IPCC para la temperatura media global. Asimismo, en las zonas rurales (figura 6) se observa un desfase de hasta 10 años en el inicio del periodo reciente de calentamiento, en comparación con zonas urbanas, para una misma región del país.

- El calentamiento y la variabilidad entre periodos cálidos y fríos es mayor, regularmente, en áreas urbanas, en comparación con las rurales. El mayor cambio de la temperatura, 


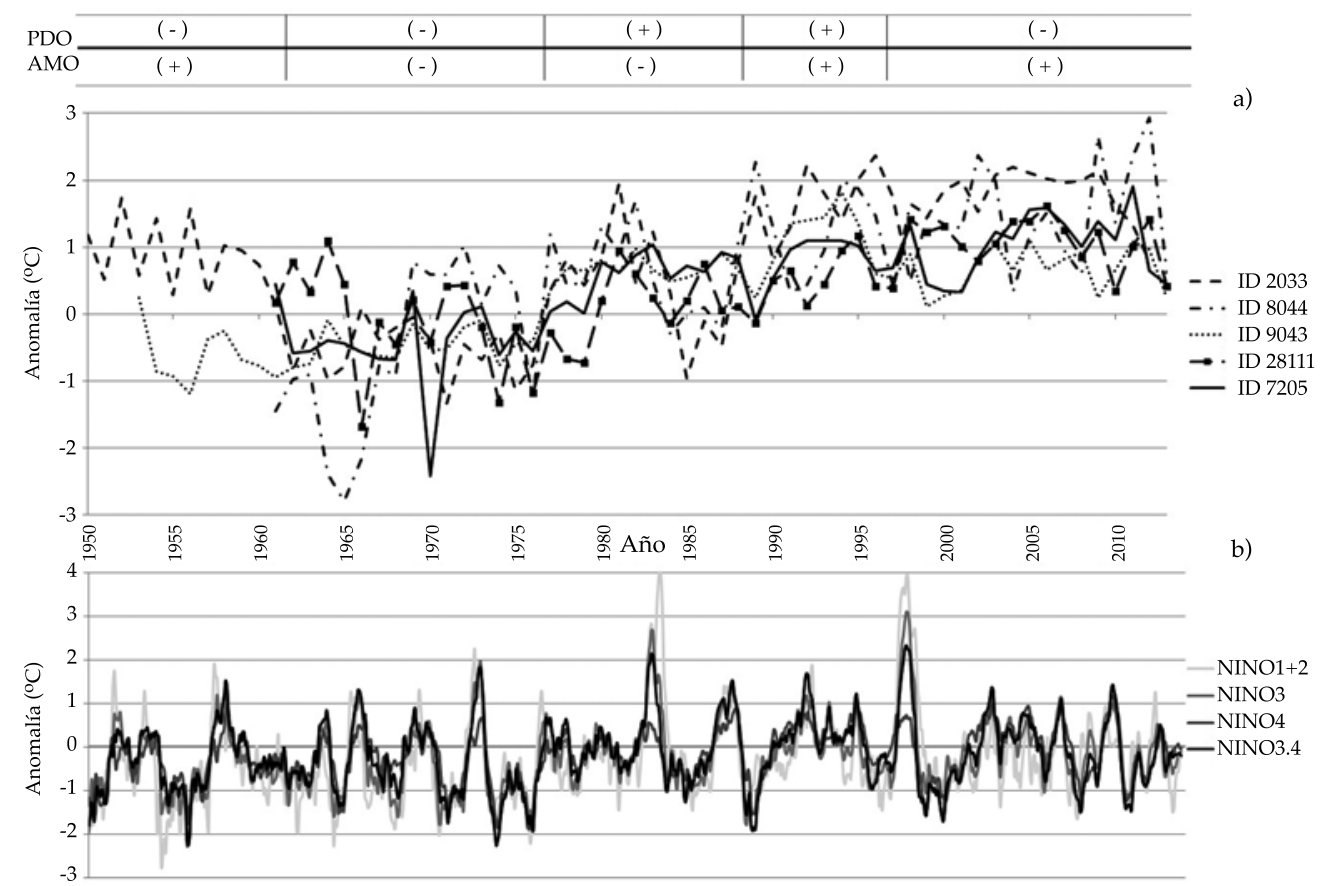

Figura 5. (a) Series de la anomalía de temperatura media anual en entornos urbanos con respecto al promedio del periodo 1961-1990. Región noroeste: estación 02033 en Mexicali, Baja California; región norte: estación 08044 en Delicias, Chihuahua; región centro: estación 09043 en Gustavo A. Madero, Ciudad de México; región Golfo de México: estación 28111 en Tampico, Tamaulipas; región Sur: estación 07205 en Comitán de Domínguez, Chiapas; (b) serie temporal de anomalías de temperatura relacionadas con El Niño y representación de las fases de la AMO y PDO. Fuente: elaborada a partir de Englehart y Douglas (2002), y de datos de NCEP disponibles en http:/ / www.cpc.ncep.noaa.gov/ data/indices/.

con valores entre 1 y $3{ }^{\circ} \mathrm{C}$, se presenta en el noroeste y en el norte, seguido por estaciones en estados del centro del país y la costa norte del Pacífico, con rangos de calentamiento entre 1 y $2{ }^{\circ} \mathrm{C}$, y con los menores cambios, menos de $1^{\circ} \mathrm{C}$, en estaciones de la costa del Golfo de México, con excepción de la ubicada en la ciudad de Tampico, Tamaulipas.

Del análisis de las tendencias de la temperatura media, con respecto al periodo base 19611990, en algunas de las estaciones de caso de las figuras 5 y 6 , con respecto a las oscilaciones de la PDO, AMO y El Niño, se identifica que:

- En la región noroeste del país, la anomalía de la temperatura en la estación urbana 02033 de Mexicali, Baja California (BC), se incrementó cuando la AMO fue positiva. En el periodo 1997-2013, el calentamiento fue mayor en comparación con el periodo 19501962, en el que se presentó una condición similar de una AMO positiva y una PDO negativa, lo que podría representar una señal de la influencia antropogénica en el clima local, no obstante que se considere la potencial influencia del efecto de isla urbana que, como lo señala el IPCC (2013), para escala global sería menor al $10 \%$ de la tendencia total. Cuando la AMO y la PDO coincidieron en su fase negativa se presentó un periodo de enfriamiento entre 1962-1977. Se identifica que El Niño tiene baja influencia en la variabilidad de la temperatura en la región.

- En la estación rural 02038, fuera de la ciudad costera de Tijuana, Baja California, 




Figura 6. (a) Series de la anomalía de temperatura media anual en entornos rurales con respecto al promedio del periodo 19611990. Región noroeste: estación 02038 en Tijuana, Baja California; región norte: estación 10021 en Inde, Durango; región centro: estación 01018 en San José de Gracia, Aguascalientes; región Golfo de México: estación 27030 en Macuspana, Tabasco; región sur: estación 12099 en Benito Juárez, Guerrero; (b) serie temporal de anomalías de temperatura relacionadas con El Niño y representación de las fases de la AMO y PDO. Fuente: elaborada a partir de Englehart y Douglas (2002), y de datos de NCEP disponibles en http:/ / www.cpc.ncep.noaa.gov/data/indices/.

se observa una influencia directa de la fase negativa de la PDO en el periodo frío registrado en la estación entre 1950-1977 probablemente debido al patrón de la fase negativa de la PDO asociado con un flujo de agua fría desde Alaska hasta la península de Baja California (Mantua, Hare, Zhang, Wallace, \& Francis, 1997). La tendencia de temperatura cambió a calentamiento posterior a 1975, coincidiendo con el cambio a la fase positiva de la PDO, calentamiento que se intensificó durante periodos intensos de El Niño, como los de 1981 y 1997. La tendencia de calentamiento se mantiene desde el año 2000 no obstante la fase negativa de la PDO, lo que probablemente represente una señal del cambio climático a escala regional.
- En la estación urbana 08044 del municipio de Delicias, Chihuahua, en el norte de México, se presentó un periodo de enfriamiento entre 1960 y 1977 probablemente asociado con la combinación de la fase negativa de la PDO y la AMO. A partir de 1977 se observa una tendencia permanente de calentamiento que coincide con la fase positiva de la PDO. De 1997 a la fecha se ha mantenido el calentamiento, no obstante que la PDO se encuentra en fase negativa, lo que podría ser compensado parcialmente por la fase positiva de la AMO y el crecimiento acelerado de la ciudad, pues la población aumentó un 60\% de 1980 a 2010.

- En el norte del país, en la estación rural 10021 en Inde, Durango, se registraron anomalías predominantemente negativas 
de la temperatura entre 1950-1992, que coinciden en la mayor parte del periodo con la fase negativa de la AMO, así como anomalías positivas puntuales probablemente relacionadas con episodios intensos de El Niño. El calentamiento reciente podría asociarse con la fase positiva de la $\mathrm{AMO}$, no obstante la fase negativa de la PDO, situación similar a la década de 1950-1960, pero ahora con signo inverso en la tendencia de la temperatura media.

- La estación urbana 09043, ubicada en un área verde en la Delegación Gustavo A. Madero, de la Ciudad de México, presentó un marcado periodo de enfriamiento entre 1953-1977, que coincidió con la fase negativa de la PDO, que predominó sobre la señal de la AMO. A partir de 1977 inició el periodo de calentamiento que se mantiene hasta 2013, mismo que presentó un máximo entre 1990 y 1995, que coincidió con la fase positiva de la PDO y la AMO, y un periodo intenso de El Niño. La señal de El Niño tiene influencia directa con el calentamiento y enfriamiento registrado en la estación. No obstante que la población en la delegación se redujo en cerca del $30 \%$, se debe considerar la influencia de la isla urbana de la Ciudad de México en la tendencia positiva de la temperatura media obtenida en la estación.
- La señal de la anomalía de la temperatura en la estación rural 01018 en San José de Gracia, Aguascalientes, en el centro del país, coincide con las fases de la AMO. La anomalía fue de enfriamiento durante la fase positiva de la AMO, entre 1950 y 1960, cálida entre 1961 y 1975 durante la fase negativa de la AMO reforzada con la fase negativa de la PDO, y fría en el periodo 1976-1987, relacionada principalmente con la potencial influencia de la fase positiva de la PDO. A partir de 1989 se inició el periodo de calentamiento marcado, que se mantuvo hasta 2013, con la probable influencia antropogénica en el clima local, no obstante que desde 1997 se tiene una combinación de las fases positiva de la PDO y AMO similar al de la década 19501960, en el que se presentó un periodo frío. La señal de El Niño incide en la anomalía anual de la temperatura y probablemente en la tendencia de calentamiento desde finales del decenio de 1990.

Como parte del análisis de la tendencia de calentamiento se generaron curvas de la función de distribución de probabilidad (FDP) para la temperatura media, ajustadas con la función de Gauss (Bidegain \& Diaz, 2011):

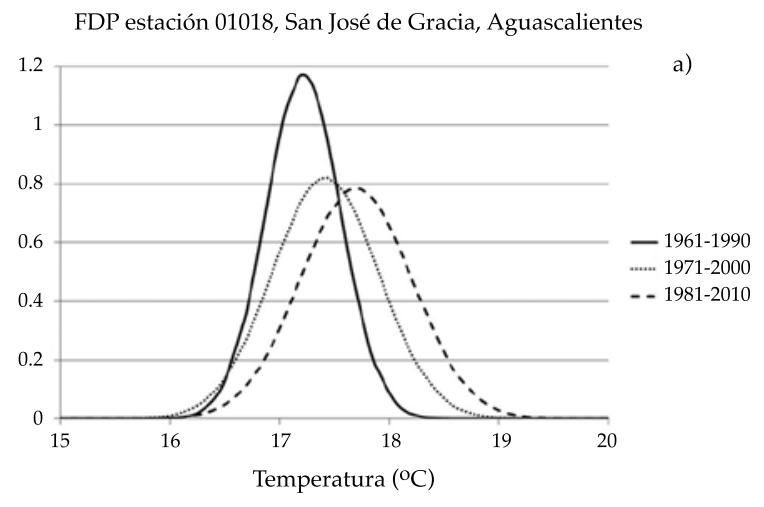

FDP estación 09043, Gustavo A. Madero, Ciudad de México

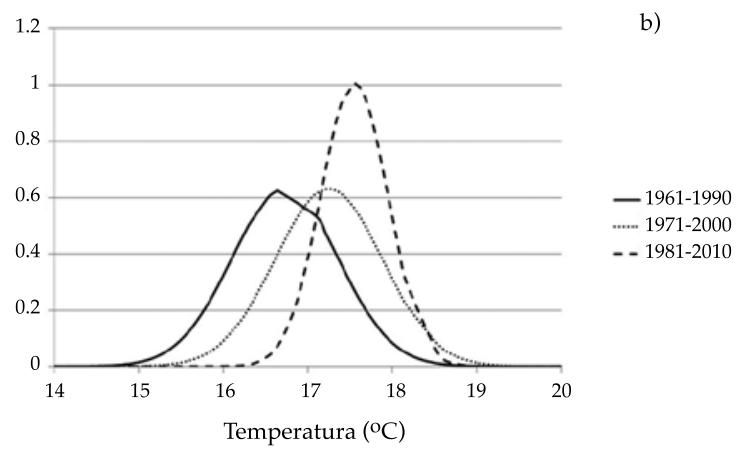

Figura 7. Curvas de la FDP de Gauss para la temperatura media en a) la estación rural 01018 en San José de Gracia, Aguascalientes, y en b) la urbana 09043 en Gustavo A. Madero, Ciudad de México. 


$$
f(x)=\frac{1}{\sigma \sqrt{2 \pi}} \exp \left[-\frac{(x-\mu)^{2}}{2 \sigma^{2}}\right], \quad-\infty<x>\infty
$$

Donde $x$ es la temperatura media, $\mu$ es el valor medio y $\sigma$ es la desviación estándar.

A partir de la curva de Gauss, calculada para tres periodos climatológicos de 30 años, se compara y analiza la evolución de la temperatura media con respecto al periodo base 1961-1990. En la figura 7 se presentan, a modo de ejemplo, los resultados para la estación rural 01018 en Aguascalientes y la estación urbana 09043 en la Ciudad de México, como casos representativos de las 20 estaciones selectas.

De la comparación de los tres periodos climatológicos de 30 años se identifica que: a) la media de la temperatura promedio se ha desplazado a valores más cálidos en ambas estaciones, con mayor incremento en la estación con entorno urbano con respecto a la rural, lo que reafirma los resultados descritos en la presente sección e indica que muy probablemente es un indicio de un cambio de clima de cada periodo; b) los máximos de la temperatura media se han desplazado hacia valores más cálidos en las dos estaciones, en tanto que los mínimos se han desplazado con mayor amplitud en la estación urbana con respecto a la rural, la primera en el orden de $1.5^{\circ} \mathrm{C}$ y la segunda en menos de $0.5^{\circ} \mathrm{C}$, y c) la variabilidad de la temperatura es mayor en los dos periodos recientes en la estación 01018, en comparación con el periodo base 1961-1990, y se ha reducido en el periodo 19812010 en la estación 09043, al estar más acotada la curva en torno a su media, con respecto a la curva del periodo base.

\section{Discusión y conclusiones}

El presente estudio muestra que las tendencias de cambio en temperatura a largo plazo definen de manera objetiva que está presente en dicha variable en nuestro país. Reafirmando la influencia de continentalidad en las estaciones climáticas analizadas, se puede asegurar que independientemente de que la influencia sea mayormente marina o continental, la señal de calentamiento global está presente.

Las 1099 estaciones, consideradas a partir de diversos conjuntos de estaciones utilizadas en estudios climáticos, conforman una base de datos climática depurada que se puede tomar como referencia, donde sólo quedaría realizar el complemento del análisis de calidad a través de la homogeneidad, proceso pendiente que representa un gran reto debido a la falta del metadato correspondiente para cada sitio. En este sentido, es recomendable que se realice en México una "antropología de metadatos", para documentar in situ y con registros históricos los cambios en las estaciones que permitan sistematizar los metadatos.

Con este estudio también se muestra que a pesar de contar con un número mayor a 5000 estaciones climáticas en el país, para estudios específicos de tendencias de temperatura este número se reduce sustancialmente a poco más de 100 estaciones irregularmente distribuidas en México, situación que conlleva a retomar el tema de la confiabilidad de la información climática y su distribución espacio-temporal.

De la distribución de 112 estaciones seleccionadas objetivamente se identifican regiones con baja cobertura en zonas del norte y sur del país, principalmente. Esta situación representa una ventana de oportunidad para fortalecer las capacidades institucionales, la infraestructura de la red de medición, el control de calidad y homogeneidad, la sistematización y difusión de la información meteorológica, climática e hidrométrica a lo largo del territorio, específicamente en las regiones que se identifican con escasa cobertura. Un beneficio directo de las mejoras será contar con insumos para una mejor gestión del riesgo de desastres ante fenómenos hidrometeorológicos y climáticos extremos, así como para la adaptación al cambio climático.

También es necesario considerar que este estudio muestra objetivamente tendencias tanto positivas como negativas, siendo las primeras mayores en una tasa de $3 / 1$, denotando que la tendencia positiva predomina; además, bajo 
este análisis se confirman las declaraciones del IPCC en el sentido de que las tendencias pueden llegar a ser diferentes, dependiendo de la latitud, longitud, topografía (Solomon et al., 2007) y ecoentorno, entre otros. En el caso de México, la interacción humana cercana a los sitios de las estaciones climáticas también es un factor por considerar. Otro factor en el que se debe realizar análisis se relaciona con los errores instrumentales en la medición, pues existe el riesgo de que las observaciones puedan parecer y comportarse como "buenos" datos, seguir rigurosamente teorías físicas válidas, y aun así conducir a conclusiones erróneas.

Asimismo, algunas estaciones con tendencia positiva y negativa están cercanas e intercaladas, lo cual tal vez esté relacionado con el entorno urbano/rural de las estaciones, diferencias en altitud, procesos físicos locales del clima y mantenimiento a la infraestructura de medición.

A partir del análisis de tendencias locales se identificó que el periodo más reciente de calentamiento intenso inicia generalmente a mediado de la década de 1970, siendo congruente con la señal identificada por el IPCC (2013) para la temperatura media global. En las zonas rurales se observa un desfase de hasta 10 años en el inicio del periodo reciente de calentamiento, en comparación con zonas urbanas para una misma región de México. El calentamiento y la diferencia entre periodos cálidos y fríos son mayores, regularmente, en áreas urbanas, en comparación con las rurales. El mayor cambio de la temperatura, con valores entre 1 y $3{ }^{\circ} \mathrm{C}$, se presenta en el noroeste y en el norte, seguido por estaciones en el centro del país y la costa norte del Pacífico, con rangos de calentamiento entre 1 y $2{ }^{\circ} \mathrm{C}$, y con los menores cambios, menor a $1{ }^{\circ} \mathrm{C}$, en la costa del Golfo de México, con excepción de la ubicada en la ciudad de Tampico, Tamaulipas.

Queda pendiente el tema de la atribución. Si bien se proponen hipótesis de algunas de sus posibles causas, es necesario realizar estudios de investigación científica, para poder determinar ya sea en forma fraccional o completa algunos de los forzantes tanto naturales como inducidos de forma completamente objetiva.

\section{Agradecimientos}

Se agradece al SMN por la entrega de la base de datos nacional climática a través del CLICOM, así como también a los doctores José Luis Bravo del CCA/UNAM y Jorge Méndez González de la UAAAN, quienes nos proporcionaron las relaciones de sus estaciones analizadas. Al Instituto Mexicano de Tecnología del Agua por el patrocinio de este trabajo $y$, en general, a todas aquellas personas e instituciones que han dedicado tiempo y recursos para mejorar la calidad de la información de la base de datos climática nacional.

\section{Referencias}

Aguilar, E. (2010). Organización de una base de datos climática de referencia para su uso en estudios de variabilidad y cambio climático en México (Fase I): uso de RSNHT.R (R-Standard Normal Homogeneity Test), v1.0. INFORME OMM/ PREMIA No. 112-03. Ciudad de México: Conagua-OMM.

Alexander, L. V., Zhang, X., Peterson, T. C., Caesar, J., Gleason, B., Klein-Tank, A., Haylock, M., Collins, D., Trewin, B., Rahimzadesh, F., Tagipour, A., Ambenje, P., Rupa-Kumar, K., Revadekar, J., Griffiths, G., Vincent, L., Stephenson, D., Burn, J., Aguilar, E., Brunet, M., Taylor, M., New, M., Zhai, P., Rusticucci, M., \& Vazquez-Aguirre, J. L. (2006). Global observed changes in daily climate extremes of temperature and precipitation. Geophys. Res. Lett., 111, D05109, 65. Recuperado de http:/ / www.knmi. $\mathrm{nl} /$ publications / fulltexts / global_extremes_for_jgr.pdf.

Bidegain, M., \& Diaz, A. (2011). Análisis estadístico de datos climáticos. Distribuciones de probabilidad. Recuperado de http://meteo.fisica.edu.uy/Materias / Analisis_ Estadistico_de_Datos_Climaticos/teorico_AEDC/ Distribuciones_Probabilidad_2011.pdf.

Bindoff, N. L., Stott, P. A., AchutaRao, K. M., Allen, M. R., Gillett, N., Gutzler, D., Hansingo, K., Hegerl, G., Hu, Y., Jain, D., Mokhov, I. I., Overland, J., Perlwitz, J., Sebbari, R., \& Zhang, X. (2013). Detection and attribution of climate change: From global to regional. In: Climate change 2013: The physical science basis. Contribution of Working Group I to the Fifth Assessment Report of the Intergovernmental Panel on Climate Change. Stocker, T. F., Qin, D., Plattner, G.-K., Tignor, M., Allen, S. K., Boschung, J., Nauels, A., Xia, Y., Bex, V., \& Midgley, P. M. (eds.). Cambridge, United Kingdom, \& New York: Cambridge University Press.

Bravo, J. L., Aspra, E., Zarraluqui, V., Gay, C., \& Estrada, F. (2012). Cluster analysis for validated climatology stations using precipitation in Mexico. Atmósfera, 25(4), 339-354. Recuperado de http://www.revistas.unam.mx/index. $\mathrm{php} / \mathrm{atm} /$ article/view/33694.

Del Valle, M. (1991). Propuesta de un índice climático para evaluar la continentalidad termopluviométrica. Lucas Mallada, 3, 129-135. 
Englehart, P. J., \& Douglas, A. V. (2002). Mexico's summer rainfall patterns: An analysis of regional modes and changes in their teleconectivity. Atmósfera, 15, 147-164. Recuperado de http://www.journals.unam.mx/index. $\mathrm{php} / \mathrm{atm} /$ article/view/8497.

Englehart, P. J., \& Douglas, A. V. (2004). Characterizing regional-scale variations in monthly and seasonal surface air temperature over México. International Journal of Climatology, 24, 1897-1909, DOI: 10.1002/ joc.1117. Recuperado de: http: / / onlinelibrary.wiley.com/ doi/10.1002/joc.1117/pdf.

Hartmann, D. L., Klein-Tank, A. M. G., Rusticucci, M., Alexander, L. V., Brönnimann, S., Charabi, Y., Dentener, F. J., Dlugokencky, E. J., Easterling, D. R., Kaplan, A., Soden, B. J., Thorne, P. W., Wild, M., \& Zhai, P. M. (2013). Observations: Atmosphere and surface. In: Climate change 2013: The physical science basis. Contribution of Working Group I to the Fifth Assessment Report of the Intergovernmental Panel on Climate Change. Stocker, T. F., Qin, D., Plattner, G. K., Tignor, M., Allen, S. K., Boschung, J., Nauels, A., Xia, Y., Bex, V., \& Midgley, P. M. (eds.). Cambridge, United Kingdom, and New York: Cambridge University Press.

Hegerl, G. C., Hoegh-Guldberg, O., Casassa, G., Hoerling, M. P., Kovats, R. S., Parmesan, C., Pierce, D. W., \& Stott, P. A. (2010). Good practice guidance paper on detection and attribution related to anthropogenic climate change (8 pp.). In: Meeting report of the Intergovernmental Panel on Climate Change Expert Meeting on Detection and Attribution of Anthropogenic Climate Change. Stocker, T. F. et al. (eds.). Bern, Switzerland: IPCC Working Group I Technical Support Unit, University of Bern.

Hegerl, G. C., Zwiers, F., Braconnot, W. P., Gillett, N. P., Luo, Y., Marengo-Orsini, J. A., Nicholls, N., Penner, J. E., \& Stott, P. A. (2007). Understanding and attributing climate change (pp. 663-745.). In: Climate Change 2007: The Physical Science Basis. Contribution of Working Group I to the Fourth Assessment Report of the Intergovernmental Panel on Climate Change. Solomon, S., Qin, D., Manning, M., Chen, Z., Marquis, M., Averyt, K. B., Tignor, M., \& Miller, H. L. (eds.). Cambridge, United Kingdom, and New York: Cambridge University Press.

INEGI (2014). Anuario Estadístico y Geográfico de los Estados Unidos Mexicanos 2013. Aguascalientes, México: Instituto Nacional de Estadística y Geografía. Recuperado de http: / / www.inegi.org.mx/prod_serv/contenidos / espanol/bvinegi / productos / integracion / pais / aeeum/2013/AEGEUM2013.pdf.

IPCC (2013). Resumen para responsables de políticas. En: Cambio climático 2013: bases físicas. Contribución del Grupo de trabajo I al Quinto Informe de Evaluación del Grupo Intergubernamental de Expertos sobre el Cambio Climático. Stocker, T. F., Qin, D., Plattner, G.-K., Tignor, M., Allen, S. K., Boschung, J., Nauels, A., Xia, Y., Bex, V., \& Midgley, P. M. (eds.). Cambridge, Reino Unido, y
Nueva York: Cambridge University Press. Recuperado de: http://www.ipcc.ch/pdf/assessment-report/ar5/ wg1/WG1_AR5_SPM_ES.pdf.

IPCC (2014). Climate change 2014. Synthesis Report Summary for Policymakers. Recuperado de: http://www.ipcc.ch/ pdf/assessment-report/ar5/syr/AR5_SYR_FINAL_ SPM.pdf.

Mantua, N. J., \& Hare, S. R. (2002). The Pacific Decadal Oscillation. Journal of Oceanography, 58, 35-44. Recuperado de: https: / / www.terrapub.co.jp/journals / JO / pdf/5801/58010035.pdf.

Mantua, N. J., Hare, S. R., Zhang, Y., Wallace, J. M., \& Francis, R. C. (1997). A Pacific interdecadal climate oscillation with impacts on salmon production. Bulletin of the American Meteorological Society, 78(6), 1069-1079.

Méndez, G. J., Ramírez, L. A., Cornejo, O. E., Zarate, L. A., \& Cavazos, P. T. (2010). Teleconexiones de la Oscilación Decadal del Pacífico (PDO) a la precipitación y temperatura en México. Boletín del Instituto de Geografía, UNAM, 73, 57-70. Recuperado de http:/ / www.journals. unam.mx/index.php/rig/article/view/ 23862.

OMM (2011). Guide to climatological practices ( $3^{\mathrm{a}}$ ed.) (117 pp.). WMO-No 100. Ginebra: OMM. Recuperado de http:/ / www.wmo.int/pages/prog/wcp/documents/Guide2. pdf.

OMM (2015). Climate Computing Project (CLICOM). Geneva: World Meteorological Organization. Recuperado de http:/ / www.wmo.int/ pages/themes/climate/ climate_ data_management_exchange.php.

Pavia, E. G., Graef, F., \& Reyes, J. (2006). Notes and correspondence. PDO-ENSO effects in climate of Mexico. Journal of Climate, 19, 6433-6438. Recuperado de http: / / journals.ametsoc.org/doi/pdf/10.1175/JCLI4045.1.

Pieyns, S. (2003). Proposal for the national hydrological and climatological reference network. Proyecto PROMMA. Informe núm. 155. Ciudad de México: CONAGUAOMM.

Semarnat-INE (2009). Cuarta Comunicación Nacional ante la Convención Marco de las Naciones Unidas sobre el Cambio Climático (274 pp.). Ciudad de México: Semarnat-INE.

Semarnat-INE (2006). Tercera Comunicación Nacional ante la Convención Marco de las Naciones Unidas sobre el Cambio Climático (254 pp.). Ciudad de México: Semarnat-INE.

Sigro, R. J. (2012). Red de referencia de estaciones climáticas de la república mexicana: asistencia técnica y capacitación para el análisis y verificación de la calidad de datos de estaciones. Informe OMM/MOMET No. 008. Ciudad de México: CONAGUA-OMM.

Solomon, S., Qin, D., Manning, M., Alley, R. B., Berntsen, T., Bindoff, N. L., Chen, Z., Chidthaisong, A., Gregory, J. M., Hegerl, G. C., Heimann, M., Hewitson, B., Hoskins, B. J., Joos, F., Jouzel, J., Kattsov, V., Lohmann, U., Matsuno, T., Molina, M., Nicholls, N., Overpeck, J., Raga, G., Ramaswamy, V., Ren, J., Rusticucci, M., Somerville, 
R., Stocker, T. F., Whetton, P., Wood, R. A., \& Wratt, D. (2007). Resumen técnico. En: Cambios climáticos 2007: base física de la ciencia. Aportes del Grupo de Trabajo I al Cuarto Informe de Evaluación del Panel Intergubernamental sobre Cambios Climáticos. Solomon, S., Qin, D., Manning, M., Chen, Z., Marquis, M., Averyt, K. B., Tignor, M., \& Miller, H. L. (eds.). Cambridge, Reino Unido, y Nueva York: Cambridge University Press.

Trenberth, K. E., Jones, P. D., Ambenje, P., Bojariu, R., Easterling, D. R., Kleint-Tank, A., Parker, D., Rahimzadeh, F., Renwick, J. A., Rusticucci, M., Soden, B., \& Zhai, P. (2007). Observations: Surface and atmospheric climate change. In: Climate Change 2007. The Physical Science Basis. Contribution of WG I to the Fourth Assessment Report of the IPCC. Cambridge, United Kingdom, and New York: Cambridge University Press.

\section{Dirección institucional de los autores}

Dr. René Lobato-Sánchez

Instituto Mexicano de Tecnología del Agua

Paseo Cuauhnáhuac 8532, Col. Progreso

62550 Jiutepec, Morelos, México

rene_lobato@tlaloc.imta.mx

M.I. Miguel Ángel Altamirano-del-Carmen

Consultor en variabilidad, cambio climático y gestión del riesgo 